\title{
Hostettler, Karin (2020): Kritik - Selbstaffirmation - Othering. Immanuel Kants Denken der Zweckmäßigkeit und die koloniale Episteme
}

Bielefeld: Transcript. 242 Seiten. 39,00€

\section{Oliver Eberl}

Angenommen: 7. April 2021 / Online publiziert: 21. April 2021

(C) Der/die Autor(en) 2021

Angesichts der intensiven Debatte um die Frage, ob Kant Rassist gewesen sei und zum Kolonialismus beigetragen habe, kommt die Dissertation der Schweizerin Karin Hostettler gerade zur rechten Zeit. Ihr gelingt etwas Außergewöhnliches: Hostettler bestätigt den Befund der kritischen Stimmen, die von einer unaufgearbeiteten Beziehung des aufklärerischen Denken Kants zum Kolonialismus ausgehen. Doch sie bietet auch jenen, die an den philosophischen Argumenten Kants interessiert sind, neue und aufschlussreiche Einsichten. Ihr Weg, diese Verbindung zu thematisieren, ist die Untersuchung der „,kolonialen Episteme“, die sich mittels „Selbstaffirmation“ und „Othering“ in der eurozentrischen Selbstpositionierung Kants ausdrückt.

Hostettler geht davon aus, dass insbesondere Kants Schriften zu den „Menschenrassen" von der Schulphilosophie übergangen oder als unphilosophisch und daher außerhalb der Philosophie stehend bewertet werden. Vonseiten der Schulphilosophie würden diese Schriften ,retrospektiv als Überschreitung der Disziplin der Philosophie eingeordnet" (S. 8) und sich so vor unbequemen Fragen gedrückt. Damit werde eine „Grenze“ installiert, die das enge disziplinäre Selbstverständnis offenbare. Es ist Hostettlers besonderer Beitrag zur Debatte, diese Grenze zu überschreiten und die inkriminierten Texte ernsthaft als philosophische Texte zu behandeln und damit auch mit der Möglichkeit zu rechnen, dass diese überhaupt einen philosophischen Sinn haben können. Das Bemühen Kants in den Aufsätzen zur „Rasse“ wird von Hostettler als „,neuartiges Denken, in dem die Natur grundsätzlich als historisch verstanden wird“ (S. 86) gedeutet. Von einer „Rasse“ spricht Kant nach Anpassung einer Gruppe von Menschen aus der Stammgattung an bestimmte klimatische Bedingungen, die dann endgültig festgelegt sind und vererbt werden. Mit diesem Befund ist es nicht mehr möglich, die Schriften als unphilosophisch auszugrenzen.

O. Eberl (四)

Leibniz-Universität Hannover, Hannover, Deutschland

E-Mail: o.eberl@ipw.uni-hannover.de 
Hostettler sieht als Inhalt der Rasseschriften europäische Überlegenheit in der Anpassung an klimatische Bedingungen und findet diese in den geschichtsphilosophischen Schriften fortgeführt (S. 100), weil hier die Subsistenzweise der Europäer durch Ackerbau als überlegen dargestellt wird. Dieser Punkt betrifft die Unterscheidungen von Jäger/Sammler-, Hirten- und Agrarvölkern (S. 92), von denen Kant Ackerbau betreibende Völker für jene mit der fortgeschrittensten Lebensweise hält (S. 109). Dieser These Hostettlers kann man nur zustimmen und sie benennt auch einen wesentlichen Punkt von Kants Eurozentrismus, der nichtstaatliche Lebensweisen tatsächlich nichts abgewinnen konnte und damit ein tiefsitzendes Vorurteil im europäischen Denken etabliert hat. Seine Beschreibung des Naturzustandes als Zustand der ,gesetzlosen Wilden“ ist nur ein Beispiel dafür.

Doch geht es Hostettler nicht allein um den Begriff der „Rasse“. Das Beweisziel liegt deutlich tiefer, nämlich in der kolonialen Episteme, die über das teleologische Denken Kants erkundet wird. „Die grundlegende These der vorliegenden Arbeit ist, dass ausgehend von den Aufsätzen zum Begriff der ,Menschenrassen“ das Denken der Zweckmäßigkeit ins Zentrum der Aufmerksamkeit gerückt werden muss“ (S. 13). Die Pointe liegt nun gerade darin, dass der Begriff „Rasse“ „ausschließlich im Rahmen des teleologischen Denkens sinnvoll verwendet werden kann“ (S. 17). Dies erklärt sich daraus, dass das Denken der Zweckmäßigkeit sich aus dem Projekt der kritischen Philosophie ergibt, das einerseits die Grenzen der Vernunft bestimmt und andererseits nach Möglichkeiten sucht, diese zu überschreiten. Die festgestellten Differenzen der „Rassen“ müssen demzufolge zweckmäßig sein. Um dies zu zeigen, greift Hostettler im Weiteren auf die geschichtsphilosophischen Schriften und die Kritik der Urteilskraft zurück.

Für ein solch komplexes Unterfangen ist das methodologische Vorgehen - und angesichts der brennenden Fragen der Debatte die daraus entwickelten Urteile - besonders interessant. Denn der Streit zwischen den unterschiedlichen Einschätzungen von Kants Schriften zu „Menschenrassen“, seiner Meinung zum Kolonialismus und Sklaverei, der Stellung Europas und der kritischen Philosophie allgemein ist ja auch eine Auseinandersetzung um den methodischen Zugriff auf diese Fragen. Hostettler entscheidet sich weder für eine ideengeschichtliche Lektüre der Teste Kants noch für eine radikale Dekonstruktion. Vielmehr sieht sie die Lösung in der Kombination von postkolonialer Kontextualisierung und Close Reading. Mit diesem Zugriff verbinden sich dann allerdings bestimmte Schwierigkeiten. Die erwähnten Stärken der Arbeit ergeben sich aus diesem Close Reading, während die Kontextualisierung eher Rückfragen provoziert.

Die postkoloniale Kontextualisierung besteht vor allem darin, ,Texte als europäische Erzeugnisse“ (S. 10) zu verstehen und dadurch das „Mitbedenken des Kolonialismus“ (S. 10) zu erreichen. Dies scheint eher einen Verdacht zu formulieren, auf den hin die Texte gelesen werden, nämlich auf den Verdacht, dass in ihnen Selbstaffirmation und Othering stattfinde, weil sie einem kolonialen Kontext zugehören. So bleiben die Aussagen Hostettlers häufig sehr allgemein. Selbstaffirmation besteht z. B. darin, „Europa als Ort der Theoriebildung“ (S. 59) zu konstruieren. Für den Kontext der „Rasse“ besteht Othering darin, dass Kant eine nochmalige Anpassung einer „Rasse“ an andere klimatische Bedingungen nicht für möglich halte. Es bleibt jenseits des Umstandes, dass Kants Beispiele einer misslungenen Neuanpassung 
kolonisierte Völker betreffen, jedoch unklar, warum die unterstellte Unmöglichkeit einer erneuten Anpassung Othering impliziert.

Überhaupt verbinden sich Unklarheiten mit der kolonialen Episteme. Foucaults Beschreibung eines Umbruchs von der traditionellen $\mathrm{zu}$ einer modernen Episteme wird knapp erläutert (S. 36-38), ohne dass klar würde, ob die moderne auch die „koloniale Episteme“ ist. Die „moderne Episteme“ nimmt eine Kluft zwischen Sprache und Natur an, sieht also jenseits der beschreibenden Elemente der Natur „ein dahinter liegendes, inneres Prinzip“ (S. 38) der Natur an, das Kant ermöglicht, die Zweckmäßigkeit als ein solches inneres Prinzip zu bestimmen. Die „koloniale Episteme" wird demgegenüber als Markieren der Positionen der Differenz innerhalb eines Systems beschrieben (S. 11).

Wenig plausibel erscheint es schließlich, in einer Arbeit über die Verbindung von Kants Denken zum Kolonialismus die Schriften zur Kritik des Kolonialismus „Zum ewigen Frieden“ und die Rechtslehre der „Metaphysik der Sitten auszusparen (S. 21). Für ein umfassendes Urteil wäre es doch wohl bedeutsam gewesen, die koloniale Episteme gegen diese Texte noch einmal zu bestätigen, statt lediglich festzustellen, dass seine Kritik am europäischen Imperialismus ,ambivalent“ (S. 101) sei. Es stimmt sicher, ,dass die interne Kritik am imperialen Europa und die Aufklärung als Zeitalter der Kritik selbst verwoben sind mit einem beständigen eurozentrischen Denken“ (S. 101). Aber der Status dieses Urteils ist uneindeutig: Was bedeutet ,verwoben“ sein? Es ist auch richtig, dass kritisch zu prüfen wäre, „ob das von Kant formulierte Ideal der kosmopolitischen Ordnung tatsächlich geeignet ist, post_koloniale Verhältnisse adäquat aufzugreifen“ (S. 116). Aber erst einmal wäre doch zu prüfen, ob dieses Ideal den damaligen kolonialen Verhältnissen in irgendeiner Form widersprochen hat und dies wiederum erscheint ohne ein Close Reading der rechtstheoretischen Schriften unmöglich. Hier wirkt es so, als würden die rechtstheoretischen und antikolonialen Schriften aus der Philosophie ausgeschlossen.

Hostettler leistet in ihrer beachtlichen Dissertation zweierlei: Sie diskutiert den Rassebegriff Kants überzeugend als naturgeschichtliche Betrachtung des Menschen aus teleologischer Perspektive, und sie problematisiert die teleologischen Prinzipien von Kants naturgeschichtlichen und geschichtsphilosophischen Schriften, die sie an die kritische Philosophie selbst zurückbindet, überzeugend als Träger eurozentrischer Selbstpositionierungen. Doch offenbart sie auch die methodischen Schwierigkeiten einer allein postkolonial kontextualisierten Lektüre klassischer philosophischer Texte.

Funding Open Access funding enabled and organized by Projekt DEAL.

Open Access Dieser Artikel wird unter der Creative Commons Namensnennung 4.0 International Lizenz veröffentlicht, welche die Nutzung, Vervielfältigung, Bearbeitung, Verbreitung und Wiedergabe in jeglichem Medium und Format erlaubt, sofern Sie den/die ursprünglichen Autor(en) und die Quelle ordnungsgemäß nennen, einen Link zur Creative Commons Lizenz beifügen und angeben, ob Änderungen vorgenommen wurden.

Die in diesem Artikel enthaltenen Bilder und sonstiges Drittmaterial unterliegen ebenfalls der genannten Creative Commons Lizenz, sofern sich aus der Abbildungslegende nichts anderes ergibt. Sofern das betreffende Material nicht unter der genannten Creative Commons Lizenz steht und die betreffende Handlung 
nicht nach gesetzlichen Vorschriften erlaubt ist, ist für die oben aufgeführten Weiterverwendungen des Materials die Einwilligung des jeweiligen Rechteinhabers einzuholen.

Weitere Details zur Lizenz entnehmen Sie bitte der Lizenzinformation auf http://creativecommons.org/ licenses/by/4.0/deed.de. 